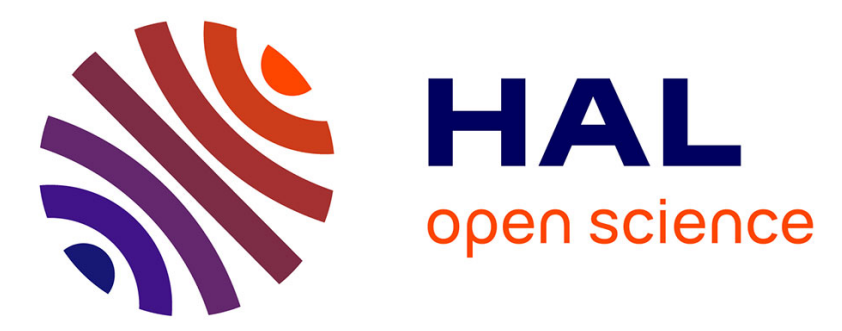

\title{
Propriétés des surfaces de GaAs (110) clivées induites par l'oxygéne et la température - contribution aux caractéristiques des diodes Schottky
}

\author{
A. Ismail, J.-M. Palau, L. Lassabatère
}

\section{- To cite this version:}

A. Ismail, J.-M. Palau, L. Lassabatère. Propriétés des surfaces de GaAs (110) clivées induites par l'oxygéne et la température - contribution aux caractéristiques des diodes Schottky. Revue de Physique Appliquée, 1984, 19 (3), pp.197-204. 10.1051/rphysap:01984001903019700 . jpa-00245183

HAL Id: jpa-00245183

https://hal.science/jpa-00245183

Submitted on 1 Jan 1984

HAL is a multi-disciplinary open access archive for the deposit and dissemination of scientific research documents, whether they are published or not. The documents may come from teaching and research institutions in France or abroad, or from public or private research centers.
L'archive ouverte pluridisciplinaire HAL, est destinée au dépôt et à la diffusion de documents scientifiques de niveau recherche, publiés ou non, émanant des établissements d'enseignement et de recherche français ou étrangers, des laboratoires publics ou privés. 


\title{
Propriétés des surfaces de GaAs (110) clivées induites par l'oxygène et la température - contribution aux caractéristiques des diodes Schottky
}

\author{
A. Ismail, J. M. Palau et L. Lassabatère \\ Laboratoire d'études des surfaces, interfaces et composants $(*)$, \\ U.S.T.L., place Eugène Bataillon, 34060 Montpellier Cedex, France
}

(Reçu le 27 juillet 1983, révisé le 2 novembre, accepté le 4 novembre 1983)

\begin{abstract}
Résumé. - Cet article est consacré à l'étude de la contribution du chauffage ou d'une exposition à l'oxygène aux propriétés des surfaces (110) de GaAs et des diodes que l'on réalise sur les surfaces ainsi modifiées. On effectue une étude des modifications du travail de sortie, de la barrière et de l'affinité électronique, étude qui permet de montrer que, dès quelques dizaines de Langmuirs des états de surfaces accepteurs et donneurs sont déjà créés et modifient la barrière de façon beaucoup plus importante, toutes proportions gardées, que le chauffage à $530^{\circ} \mathrm{C}$. A plus forte exposition $\left(2 \times 10^{4}\right.$ Langmuirs) les topographies sont uniformes et l'action des défauts de clivage sur le travail de sortie et le photovoltage, est estompée. Les diodes obtenues par dépôt d'argent sur ces surfaces ont des propriétés légèrement différentes de celles obtenues par dépôt d'argent sur la surface aussitôt après clivage. Ces différences sont analysées en termes de défauts, de dipôle et de perturbation de l'interface induite par l'oxygène ou le chauffage.

Abstract. - The first part of this paper is devoted to the study of the GaAs (110) surface properties, to their modification by $\mathrm{O}_{2}$ exposure and by annealing. By using contact potential difference topographies (TCPD), surface photovoltage topographies (TSPV), we show that oxygen exposure as low as a few tens of Langmuirs induces barrier modifications (due to donor and acceptor state formation) and affinity variations as large as few hundred millielectronvolts. The topographies get uniform, the cleavage defect are erased by $2 \times 10^{4}$ Langmuirs. Annealing up to $530^{\circ}$ also gives barrier and affinity modification. Diodes are then achieved by depositing $\mathrm{Ag}$ on these surfaces and we compare their properties as deduced from $I(V) C(V)$ curves, to the properties of diodes achieved on cleaved surface without any other treatment. Oxygen exposure induces higher modifications than annealing which are analysed in terms of sharpness and structure of the interface.
\end{abstract}

\section{Introduction.}

$\mathrm{Du}$ fait de l'importance technologique du contact métal-semiconducteur et de son caractère élémentaire qui lui confère une remarquable utilité pour l'étude des mécanismes physiques, physicochimiques ou électroniques, des études nombreuses lui ont été consacrées ces dernières années. Ces études ont conduit à admettre que la structure physique d'une diode Schottky est beaucoup plus complexe que la structure idéale initialement envisagée et que des défauts à la surface ou près de la surface du semiconducteur, des réactions chimiques, pouvaient dans de nombreux cas contribuer fortement à la formation de la diode et à ses propriétés [1-8]... Ces conclusions ont orienté les travaux vers une meilleure appréhension des interactions au niveau de la couche de surface, mais aussi des couches sous-jacentes et ont conduit à réexaminer tous les résultats expérimentaux acquis à partir de

(*) U.R. No 1022 associée au C.N.R.S. concepts nouveaux. Parmi ces concepts celui des défauts proposé par Spicer et al. [1] occupe une place particulière en raison de sa généralité et du large éventail de ses possibilités d'application. Selon ces auteurs, des défauts spécifiques du semiconducteur présents à la surface avant tout traitement ou induits par une action extérieure, en déterminent les propriétés et contribuent de façon prépondérante à la formation du contact. Ce sont les états correspondants qui seraient à l'origine de l'ancrage du niveau de Fermi qui est caractéristique des composés III-V.

Si ce modèle rend bien compte de certains résultats, il ne peut expliquer l'ensemble des faits expérimentaux car il ne prend pas en compte la relaxation de la surface, la modification de sa nature chimique, l'action spécifique éventuelle du métal. Il suppose, en particulier, que cette relaxation n'est pas perturbée par le dépôt ce qui n'est pas établi à l'heure actuelle de façon certaine. Au cas où il en serait autrement $[9,10]$, les états intrinsèques en réintégrant la bande interdite, conduiraient à l'ancrage du niveau de Fermi ce qui 
expliquerait aussi certains résultats expérimentaux. Cette dernière hypothèse bien que discutée en particulier par R. H. Williams [11] sert à l'heure actuelle de support à des travaux qui jettent un éclairage nouveau sur les problèmes de l'interaction métal-gazsemiconducteur $[12,13]$. Comment dans ces conditions appréhender l'influence d'une atmosphère contrôlée ou de la température sur les propriétés de surface ou d'interface ? Différents auteurs qui se sont attachés à répondre à cette question ont montré que l'interaction gaz semiconducteur se traduisait par la formation d'états extrinsèques qui peuvent comme c'est le cas lors de l'interaction $\mathrm{O}_{2}-\mathrm{GaSb}, \mathrm{GaAsInP}$ [14-16] ancrer le niveau de Fermi. Elle peut conduire à une modification notable de l'ordre et à la formation d'un composé nouveau dont la présence contribuerait fortement aux propriétés du contact [17].

La température quant à elle permet de réorganiser la surface, d'en modifier éventuellement la structure ou la relaxation, d'en perturber la composition par la création de lacunes, lacunes dont le rôle est essentiel dans le modèle de Spicer.

Quelle est la contribution de ces effets aux propriétés du contact ? Les études tendant à répondre à cette question sont relativement fragmentaires et ponctuelles. C'est ainsi que si l'on sait que le chlore désordonne la surface de l'InP et diminue la barrière des diodes [4], que l'oxygène peut sur GaAs conduire à des effets comparables [5], il n'est pas à ce jour de réponse globale claire. Il en est de même en ce qui concerne la contribution du chauffage qui n'a donné lieu qu'à des études peu nombreuses de la composition en surface pour des températures élevées [18], des propriétés électroniques [19]. De façon générale donc, la recherche d'une corrélation entre propriétés de surface et propriétés des diodes s'avère encore nécessaire et les quelques travaux publiés à ce jour [20-26] ne sont pas à même d'apporter une réponse aux questions posées.

Le travail que nous présentons dans cet article doit être situé dans ce contexte. Nous étudions l'action de l'oxygène et de la température sur les propriétés de surface de GaAs (travail de sortie, photovoltage) dans la gamme des expositions inférieures à $10^{5}$ Langmuirs et des températures inférieures à $550^{\circ} \mathrm{C}$. Nous élaborons des diodes sur la surface ainsi perturbée, diodes dont nous comparons les propriétés à celles des diodes réalisées sur surface non perturbée.

\section{Action de l'oxygène et de la température.}

Nous nous limiterons aux éléments nécessaires pour appréhender les résultats que nous présentons et les situer dans le contexte général.

2.1 ACTION DE L'OXYGÈNE. - Les questions qui se posent lors de l'action de l'oxygène sur la surface de GaAs, action dont un excellent bilan a été récemment présenté par W. Ranke et K. Jacobi [30] C. Y. Su et al.
[31] peuvent être résumés comme suit :

- l'oxygène s'adsorbe-t-il sur $\mathrm{Ga}$, sur As, sur les deux ?

- quelle est la nature et la distribution des liaisons?

- quelles sont les caractéristiques essentielles de l'adsorption ? domaines d'exposition correspondant à des modifications mesurables par les techniques actuelles, cinétique d'adsorption, coefficients de collage...

- quel est le rôle des défauts initiaux de surface, de l'état de l'oxygène (excité ou non), de la température, de la stimulation électronique...?

- quelles perturbations induites peut-on envisager ? Comment peuvent-elles intervenir au niveau des propriétés des diodes ? Certaines de ces questions ont reçu un début de réponse. A partir de mesures utilisant le rayonnement du synchrotron [15, 31-34] Spicer $e t$ al. avaient jusqu'à ces dernières années conclu que l'oxygène se liait seulement à As et ce pour des expositions allant jusqu'à $10^{12}$ Langmuirs. Ludeke [35], quant à lui, à partir de spectres de perte d'énergie d'électrons concluait à des possibilités de liaison de l'oxygène avec $\mathrm{Ga}$ et As. Les résultats de Brundle et Seybold [36] allaient dans ce sens et permettaient à ces auteurs de conclure contrairement à Mele et Joannopoulos [37] que l'oxygène était dissocié. Tout dernièrement en réponse à ces conclusions divergentes, C. Y. Su et al. [31] ont proposé un modèle suivant lequel l'oxygène formerait des liaisons du type $\mathrm{As}=\mathrm{O}$ et des liaisons pontées du type $\mathrm{Ga}-\mathrm{O}-$ As.

Ces différentes conclusions ont été déduites essentiellement de résultats expérimentaux obtenus pour la plupart dans le domaine des expositions supérieures à $10^{6}$ Langmuirs. A plus faible exposition les résultats sont moins nombreux et parfois contradictoires. En effet si, P. E. Gregory et al. [38] ont, en 1976, mis en évidence certains cas particuliers de modification des courbes de photoémission et de position du niveau de Fermi pour des expositions supérieures à $10^{4}$ Langmuirs, Luth et al. [39], puis Pianetta et al. [15] présentaient peu après des résultats mettant en évidence respectivement une formation de barrière pour des expositions inférieures à 1 Langmuir et des modifications de courbes de photoémission pour des expositions supérieures à 1 Langmuir. En 1979, C. D. Thault et al. [40] localisaient une bande d'états induits par l'oxygène à 1 Langmuir bande interprétée par une relaxation partielle de la reconstruction de surface ce qui conduisait à envisager l'action de l'oxygène en termes de modification de structure de surface, voire d'ordre et de désordre.

\subsection{ACTION DE LA TEMPÉRATURE. - La température} peut induire des variations de stœchiométrie par départ non congruent de ses composants et des variations de structure. Les modifications de stœchiométrie ont donné lieu à des travaux poussés [18, 41, 42] qui ne permettent cependant pas de connaître les 
densités de lacunes en surface dans le domaine des températures inférieures à $500^{\circ} \mathrm{C}$. Cette densité s'avère trop faible pour qu'elle puisse être mesurée par les techniques habituelles. Elle peut être cependant suffisante pour induire des modifications mesurables des propriétés électroniques [19, 43, 44]. Selon ces auteurs $[19,44]$ il y aurait en particulier une évolution importante du travail de sortie lorsque la température atteint $350^{\circ} \mathrm{C}$, évolution qui résulterait de l'apparition d'états situés au voisinage du milieu de la bande interdite. Cette évolution conduit à envisager la contribution des lacunes aux propriétés de la surface puis des diodes et ce, entre autres, dans le cadre du modèle de Spicer.

\section{Méthode expérimentale.}

Toutes les études sont effectuées in situ dans un ensemble permettant de travailler sous $10^{-10}$ Torr [25] au cours d'un seul cycle. Les échantillons de type n et $p$ se présentent sous la forme de parallélépipèdes de $4 \times 8 \mathrm{~mm}^{2}$ de section droite. Ils sont clivés et étudiés par paires (un échantillon n et un p). Pour cela, ils sont présentés en face des différents postes d'étude qui permettent des relevés topographiques de la différence de potentiel de contact entre le semiconducteur et une électrode de référence (TCPD), des relevés de topographie de photovoltage (TSPV) et de composition (AES). La température de l'échantillon peut être ajustée entre $100 \mathrm{~K}$ et $800 \mathrm{~K}$ environ sans que le vide soit modifié.

L'oxygène pur est introduit par une microfuite. On établit entre la vitesse d'entrée de gaz et la vitesse de pompage un équilibre de façon à obtenir la pression désirée, pression comprise suivant les cas entre $2 \times 10^{-8}$ Torr et $4 \times 10^{-6}$ Torr. Après exposition, effectuée jauge éteinte, on rétablit le vide limite et effectue les différentes études de surface. On dépose ensuite des plots de métal de diamètre voisin du $\mathrm{mm}$ par évaporation à très faible vitesse de façon à obtenir des fractions de monocouches. On étudie après chaque dépôt les propriétés de la surface, puis on achève les diodes et on les caractérise par le relevé des courbes $C(V)$ et $I(V)$.

\section{Résultats.}

Les études que nous avons effectuées ont porté sur des échantillons fournis par MCP dont les caractéristiques sont les suivantes :

type n-dopé Si $n=7,5 \times 10^{16} \mathrm{~cm}^{-3} E_{\mathrm{c}}-E_{\mathrm{f}} \approx 60 \mathrm{meV}$ type p-dopé $\mathrm{Zn} p=5,7 \times 10^{16} \mathrm{~cm}^{-3} E_{\mathrm{f}}-E_{\mathrm{v}} \approx 140 \mathrm{meV}$.

Les résultats présentés de façon globale, tableau I, $I^{\prime}$, II ont été obtenus à partir du relevé des topographies TCPD, TSPV et des courbes $C(V), I(V)$.

4.1 ACTION DE LA TEMPÉRATURE. - La figure 1 représente les topographies du travail de sortie $\phi$ d'échantillons de type $n$ et $p$ obtenues après clivage et après chauffage à $420^{\circ} \mathrm{C}$ pendant $20 \mathrm{~min}$. L'étude de ces topographies et du photovoltage montre que :

- le travail de sortie du type $\mathrm{p}$ est pratiquement uniforme $[25,27]$; les défauts de clivage ont un effet peu important; le photovoltage et la barrière sont pratiquement nuls sur zone bien clivée, de quelques dizaines de millivolts sur zone avec défauts;

- le travail de sortie du type n n'est pas uniforme. Il existe des zones de défauts caractérisées par un photovoltage et une barrière de surface importants de plusieurs centaines de millivolts et des zones bien clivées avec une barrière très faible;

- la différence des travaux de sortie des zones bien clivées des types $\mathrm{p}$ et $\mathrm{n}$ est voisine de la distance énergétique entre les niveaux de Fermi en volume $E_{\mathrm{Fn}}-E_{\mathrm{Fp}}$

Tableau I. - Travail de sortie et photovoltage de GaAs (110) clivés après recuit, adsorption d'oxygène et dépôt de $0,25 \mathrm{mc} \mathrm{Ag.}$

[Work function and SPV of cleaved (110) GaAs after annealing, oxygen adsorption and $0.25 \mathrm{ml} \mathrm{Ag} \mathrm{deposit.]}$

GaAs (110)

\begin{tabular}{|l|r|r|r|r|r|r|r|r|}
\cline { 2 - 9 } \multicolumn{1}{c|}{} & $\begin{array}{r}\text { Recuit } \\
420^{\circ} \mathrm{C}\end{array}$ & $\begin{array}{c}\text { Recuit } \\
530^{\circ} \mathrm{C}\end{array}$ & $\begin{array}{c}100 \mathrm{~L} \\
\text { oxygène }\end{array}$ & $\begin{array}{c}2 \times 10^{4} \mathrm{~L} \\
\text { oxygène }\end{array}$ & $\begin{array}{c}\text { Recuit } \\
530^{\circ} \mathrm{C} \\
+1 / 4 \mathrm{Ag}\end{array}$ & $\begin{array}{c}100 \mathrm{~L} \\
\text { (oxygène) } \\
+1 / 4 \mathrm{Ag}\end{array}$ & $\begin{array}{c}2 \times 10^{4} \mathrm{~L} \\
\text { (oxygène) } \\
+1 / 4 \mathrm{Ag}\end{array}$ & $\begin{array}{c}\text { Clivée } \\
\text { propre } \\
+1 / 4 \mathrm{Ag}\end{array}$ \\
\hline$\phi_{0 \mathrm{p}}-\phi_{\mathrm{p}} \pm 50 \mathrm{meV}$ & 250 & 550 & 450 & 520 & 880 & 720 & 850 & 850 \\
\hline$\phi_{\mathrm{n}}-\phi_{0 \mathrm{n}} \pm 50 \mathrm{meV}$ & 550 & 350 & 420 & 420 & 300 & 300 & 280 & 300 \\
\hline$\phi_{\mathrm{p}}-\phi_{\mathrm{n}} \pm 50 \mathrm{meV}$ & 400 & 300 & 330 & 230 & 20 & 150 & 90 & 50 \\
\hline $\mathrm{SP} V_{\mathrm{n}} \pm 20 \mathrm{mV}$ & -200 & -170 & -190 & -330 & -240 & -320 & -390 & -240 \\
\hline $\mathrm{SP} V_{\mathrm{p}} \pm 10 \mathrm{mV}$ & 10 & 40 & 50 & 70 & 30 & 50 & 10 & 30 \\
\hline
\end{tabular}


Tableau I'. - Potentiel de surface $V_{\mathrm{s}}$ de ĠaAs (110) clivé après recuit et adsorption d'oxygène et modification d'affinité électronique $\Delta \chi$ induites par ces traitements.

[Surface potential $V_{\mathrm{S}}$ on cleaved (110) GaAs after annealing and after oxygen adsorption and electronic affinity modifications resulting from these treatments.]

\begin{tabular}{|c|r|r|r|r|}
\cline { 2 - 5 } \multicolumn{1}{c|}{} & $\begin{array}{c}\text { Recuit } \\
420^{\circ} \mathrm{C}\end{array}$ & $\begin{array}{c}\text { Recuit } \\
530^{\circ} \mathrm{C}\end{array}$ & $\begin{array}{c}100 \mathrm{~L} \\
\text { oxygène }\end{array}$ & $\begin{array}{c}2 \times 10^{4} \mathrm{~L} \\
\text { oxygène }\end{array}$ \\
\hline$V_{\mathrm{Sn}} \pm 50 \mathrm{mV}$ & -660 & -600 & -600 & -640 \\
\hline$V_{\mathrm{Sp}} \pm 50 \mathrm{mV}$ & 140 & 300 & 270 & 330 \\
\hline$\Delta \chi \pm 50 \mathrm{meV}$ & -110 & -250 & -180 & -220 \\
\hline
\end{tabular}
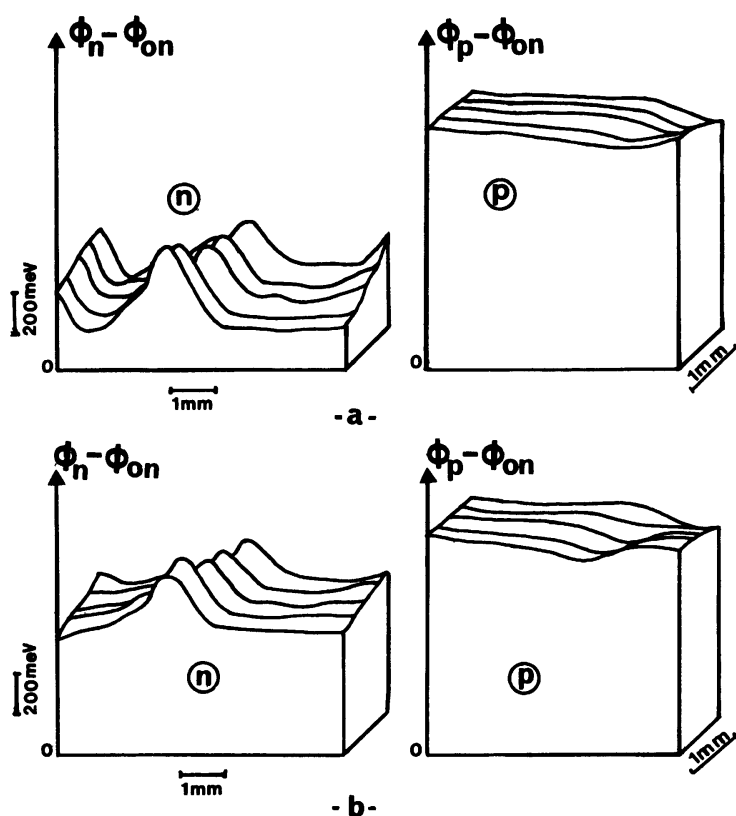

Fig. 1. - Topographies du travail de sortie relevées sur une partie de l'échantillon de GaAs (110) de type $\mathrm{n}$ et $\mathrm{p}: \mathrm{a}-$ surfaces initiales. $\mathrm{b}-$ surfaces après recuit à $420^{\circ} \mathrm{C}\left(\phi_{0 \mathrm{n}}\right.$ travail de sortie de la surface initiale de type $n$ en l'absence de barrière).

[Partial work function topographies of $\mathrm{n}$ and $\mathrm{p}$ types (110) GaAs samples (origin is $\phi_{\mathrm{n}}$ for the $\mathrm{n}$ type in flat band condition) : a - before annealing. $b$ - after annealing at $420^{\circ} \mathrm{C}$.]

- le recuit à $420^{\circ} \mathrm{C}$ tend à uniformiser le travail de sortie et le photovoltage de type $\mathrm{n}$, à rapprocher les travaux de sortie des échantillons des deux types tout en maintenant entre eux une différence de $400 \mathrm{mV}$. Par ailleurs, photovoltage et barrière sur type $p$ restent négligeables;

- le recuit à $530^{\circ} \mathrm{C}$ perturbe de façon beaucoup plus importante les topographies (Fig. 2). Il se traduit par l'apparition d'un photovoltage uniforme sur type $\mathrm{p}$ correspondant à l'apparition de donneurs par l'uniformisation du photovoltage et des variations
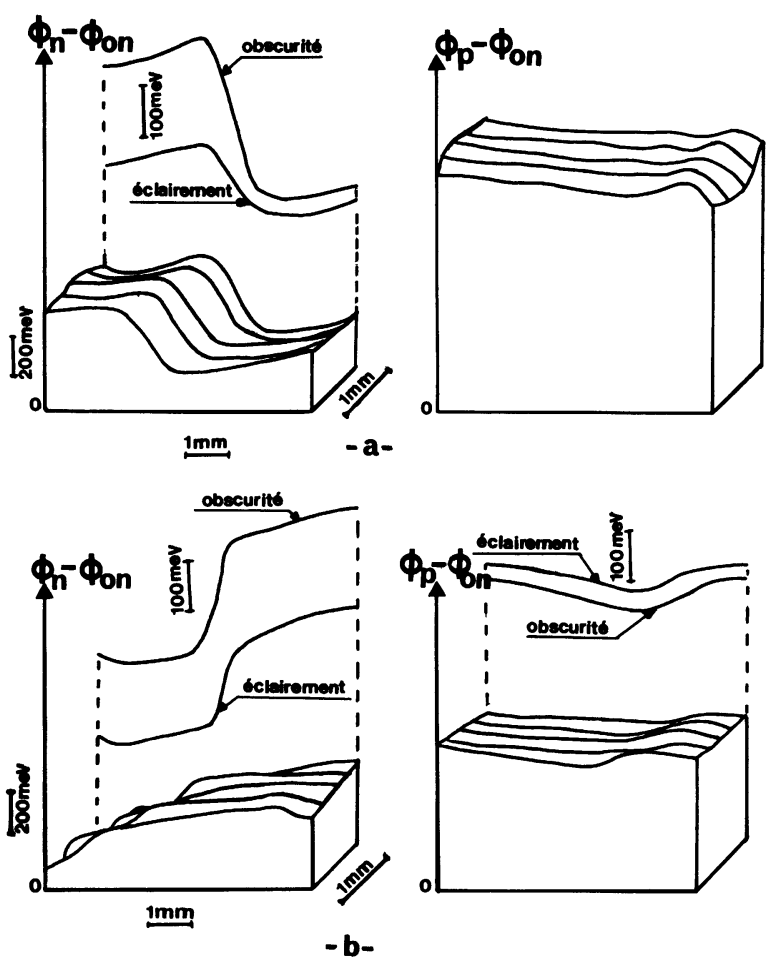

Fig. 2. - Topographies du travail de sortie relevées sur une partie de l'échantillon de GaAs (110) de type $\mathrm{n}$ et $\mathrm{p}: \mathrm{a}-$ surfaces initiales. $\mathrm{b}-$ surfaces après recuit à $530^{\circ} \mathrm{C}\left(\phi_{0 \mathrm{n}}\right.$ travail de sortie de la surface initiale de type $n$ en l'absence de barrière).

[Partial work function topographies of $n$ and $p$ types (110) GaAs samples : $a$ - before annealing. $b$ - after annealing at $\left.530^{\circ} \mathrm{C}.\right]$

importantes de $\phi$ sur type $\mathrm{n}$. La différence entre travaux de sortie des zones mal clivées et bien clivées décroît et peut même dans certains cas s'inverser (Fig. 2).

4.2 ACTION DE L'OXYGÈnE. - Sous l'action de $\mathrm{O}_{2}$ les topographies tendent à s'uniformiser (Fig. 3). L'évolution des travaux de sortie $\phi_{\mathrm{n}}$ et $\phi_{\mathrm{p}}$ et du photo- 
Tableau II. - Hauteur de barrière $\phi_{\mathrm{B}}$, facteur d'idéalité $n$ et potentiel de diffusion $V_{\mathrm{D}}$ des diodes Schottky $\mathrm{Ag} / \mathrm{GaAs}$ (110) clivé réalisées sur différentes surfaces.

[Schottky barrier height $\phi_{\mathrm{B}}$, ideality factor $n$ and diffusion potential $V_{\mathrm{D}}$ of $\mathrm{Ag} / \mathrm{GaAs}$ (110) Schottky diodes achieved on different surfaces.]

GaAs (110) clivée

\begin{tabular}{|lc|c|c|c|c|c|}
\cline { 2 - 6 } \multicolumn{1}{c|}{} & $\begin{array}{c}\text { Surface recuite } \\
\text { à } 420^{\circ} \mathrm{C}\end{array}$ & $\begin{array}{c}\text { Surface recuite } \\
\text { à } 530^{\circ} \mathrm{C}\end{array}$ & $\begin{array}{c}100 \mathrm{~L} \\
\text { oxygène }\end{array}$ & $\begin{array}{c}2 \times 10^{4} \mathrm{~L} \\
\text { oxygène }\end{array}$ & $\begin{array}{c}\text { Surface } \\
\text { propre }\end{array}$ \\
\hline $\begin{array}{l}\phi_{\mathrm{Bn}} \\
I-V\end{array}$ & $\begin{array}{c}\text { type } \mathrm{n} \\
(\mathrm{meV})\end{array}$ & 860 & 860 & 860 & 850 & 840 \\
\hline $\begin{array}{l}\text { type p } \\
\mathrm{meV}\end{array}$ & 435 & 430 & 445 & 440 & 380 \\
\hline$n$ & $\begin{array}{l}\text { type n } \\
\text { type p }\end{array}$ & 1,08 & 1,08 & 1,10 & 1,07 & 1,14 \\
\hline$C-V$ & $\begin{array}{l}V_{\mathrm{Dn}} \\
\mathrm{mV}\end{array}$ & 870 & 1,27 & 1,33 & 1,9 & 1,50 \\
\hline
\end{tabular}
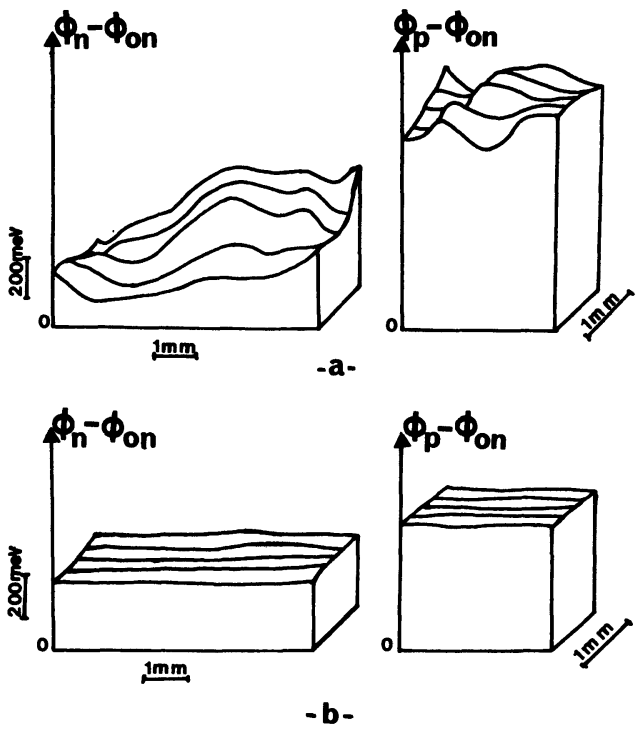

Fig. 3. - Topographies du travail de sortie relevées sur une partie de l'échantillon de GaAs (110) de type $n$ et $\mathrm{p}: \mathrm{a}-$ surfaces initiales. $\mathrm{b}-$ surfaces après exposition à $2 \times 10^{4}$ Langmuirs (oxygène) ( $\phi_{0 \mathrm{n}}$ travail de sortie de la surface initiale de type $n$ en l'absence de barrière).

[Partial work function topographies of $\mathrm{n}$ and $\mathrm{p}$ types (110) GaAs samples : a - initial. $\mathrm{b}-$ after exposition to $2 \times 10^{4}$ Langmuirs of oxygen.]

voltage dans les zones bien clivées sont représentées figure 4. Dans la gamme 0-200 Langmuirs environ, $\phi_{\mathrm{n}}$ et $\phi_{\mathrm{p}}$ varient régulièrement avec l'exposition ( $\phi_{\mathrm{p}}$ décroît, $\phi_{\mathrm{n}}$ croît) et le photovoltage, qui correspond dans tous les cas à une zone de déplétion, augmente. Entre 200 Langmuirs et $2 \times 10^{4}$ Langmuirs, les REVUE DE PHYSIQUE APPLIQUÉE. - T. 19, No 3, MARS 1984

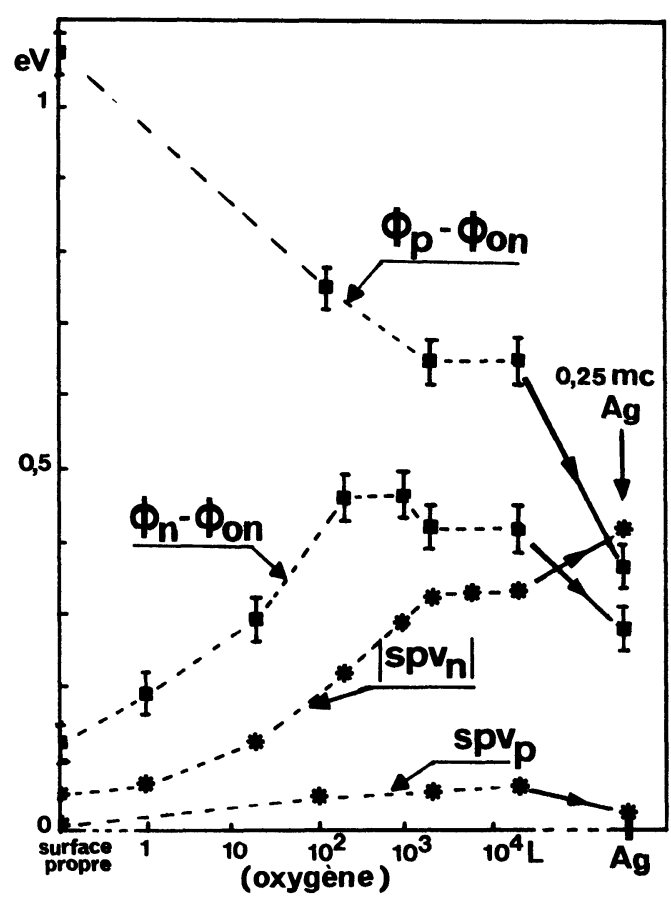

Fig. 4. - Evolution du travail de sortie et du photovoltage de GaAs (110) de type $n$ et $\mathrm{p}\left(\phi_{\mathrm{n}}, \phi_{\mathrm{p}}, \operatorname{SP} V_{\mathrm{n}}, \operatorname{SP} V_{\mathrm{p}}\right)$ après exposition à l'oxygène et après dépôt de 0,25 monocouche d'argent.

[Modifications of the work function and the surface photovoltage due to oxygen exposure and to deposition of 0.25 monolayer of silver.]

travaux de sortie varient peu. La différence $\phi_{\mathrm{p}}-\phi_{\mathrm{n}}$ reste de l'ordre de $250 \mathrm{mV}$-ce qui correspond seulement à un début d'ancrage de $E_{\mathrm{F}}^{\prime}$. 
4.3 Dépôt métalliQue. - Sous l'action d'un quart de monocouche d'argent, toutes les topographies deviennent uniformes. Dans le cas des surfaces préalablement soumises à 100 Langmuirs $\mathrm{O}_{2} \phi_{\mathrm{p}}$ et $\phi_{\mathrm{n}}$ diminuent, le photovoltage sur type $\mathrm{p}\left(\mathrm{SPV}_{\mathrm{p}}\right)$ reste identique à lui-même, le photovoltage sur type $n$ $\left(\mathrm{SVP}_{\mathrm{n}}\right)$ croît fortement. $\phi_{\mathrm{p}}-\phi_{\mathrm{n}}$ est faible. Le niveau de Fermi est pratiquement ancré en surface.

4.4 Diodes. - Les résultats les concernant sont portés tableau II.

Les valeurs numériques conduisent à quelques analyses générales :

- la barrière déduite des courbes $I(V)$ est pratiquement indépendante du traitement sur type $\mathrm{n}$ alors que sur type $p$ elle est augmentée par le chauffage ou l'adsorption d'oxygène ;

- le facteur d'idéalité est modifié de façon notable par le traitement de surface tout comme la hauteur de barrière déduite des courbes $C(V)$.

\section{Discussion.}

L'action de l'oxygène et des métaux sur les surfaces de GaAs ou d'InP et les modifications de propriétés des diodes qui en résultent ont été fréquemment interprétées jusqu'à ces dernières années en terme de désordre ou de diffusion. Dans le cas GaAs, il semblait que, indépendamment du métal, de sa réaction avec la surface, l'ancrage du niveau de Fermi se faisait toujours au même endroit. C'est ce fait qui avait conduit Spicer et al. à suggérer que cet ancrage avait pour origine des défauts induits indirectement par le métal, et, l'état de désordre ayant été expérimentalement mis clairement en évidence, à introduire la notion de défauts.

Des études récentes se sont proposées de préciser ces points et de rechercher de façon plus fine la contribution de la structure de la surface de la stœchiométrie.

W. 'I. Wang [28] partant de surfaces différemment reconstruites a mis en évidence des variations de la barrière Schottky dans les diodes GaAs-Al pouvant atteindre $100 \mathrm{meV}$.Z. M. Lu et al. [21] ont, quant à eux, montré que l'interaction de la surface de GaAs avec $\mathrm{Au}$ était sensiblement modifiée par une exposition préalable de celle-ci à l'oxygène. Pour des recouvrements correspondant à une petite fraction de monocouche seulement l'interdiffusion de l'or serait notablement perturbée et la position de l'ancrage déplacée. L'amorphisation de la surface résultant d'exposition allant de $10^{5}$ à $10^{9}$ Langmuirs $\left(\mathrm{O}_{2}\right)$ diminuerait encore la contribution de l'or aux propriétés de l'interface.

Dans le cas de notre étude, la modification de la surface et de ses propriétés électroniques est déjà très nette à faible exposition. A 1 Langmuir $\left(\mathrm{O}_{2}\right)$, et comme précédemment noté par d'autres auteurs $[39,40]$ travaux de sortie et barrières sont appréciablement changés. La densité de charge dans les états accepteurs et donneurs créés par l'interaction croît jusqu'à des expositions de quelque $10^{3}$ Lang- muirs exposition pour laquelle le niveau de Fermi et le photovoltage se stabilisent. A $2 \times 10^{4}$ Langmuirs les topographies sont parfaitement uniformes et les barrières estimées à partir de mesures du photovoltage sont de plusieurs centaines de millivolts.

L'adsorption s'est donc traduite par une formation de barrière et par des modifications d'affinité électronique que l'on peut déduire des topographies. On sait en effet que les hauteurs de barrière $V_{\mathrm{Sn}}$ et $V_{\mathrm{Sp}}$ dans les échantillons $n$ et $p$ ayant subi le même traitement, sont données par la relation

$$
\phi_{\mathrm{p}}-\phi_{\mathrm{n}}=E_{\mathrm{Fn}}-E_{\mathrm{Fp}}+q V_{\mathrm{Sn}}-q V_{\mathrm{Sp}}
$$

relation qui suppose une même affinité électronique $\chi$ sur les deux échantillons.

Les valeurs expérimentales fournissent donc $V_{\mathrm{Sp}}-V_{\mathrm{S}}$. Moyennant l'hypothèse, qui semble expérimentalement acceptable en première approximation, que les courbes de variations de $\operatorname{SP} V_{n}$ et $\operatorname{SP} V_{\mathrm{p}}$ en fonction de la barrière après modification de la surface se déduisent de courbes caractéristiques de la surface initiale par une homothétie de même rapport, on peut déduire les hauteurs des barrières de surface de $V_{\text {Sp }}-V_{\text {Sn }}$ et du photovoltage.

Les variations éventuelles de $\chi$ s'obtiennent ensuite directement à partir de $\phi_{\mathrm{n}}-\phi_{0 \mathrm{n}}, \phi_{\mathrm{p}}-\phi_{0 \mathrm{p}}$. Les données ainsi obtenues portées tableau $I^{\prime}$ ne sont qu'indicatives en raison de l'approximation que nous avons faite. Elles constituent cependant des ordres de grandeurs raisonnables et mettent en évidence une décroissance de $\chi$ sous l'action de $\mathrm{O}_{2}$. Comme précédemment noté sur certains échantillons par P. E. Gregory et al. [38], ceci peut s'interpréter par la formation d'un dipôle obtenu par transfert d'électrons des atomes de surface vers des atomes d'oxygène qui se seraient placés juste sous la surface ou par des modifications de contraintes se traduisant par un réarrangement de surface induisant une modification de $\chi$. Un déplacement d'atomes de $\mathrm{Ga}$ et d'As vers leur position non relaxée se traduirait, en effet, par une modification de $\chi$ de même sens que celle mesurée. Le fait que les zones perturbées lors du clivage soient totalement uniformisées au plan de la barrière comme de l'affinité va plutôt dans le sens de la deuxième hypothèse et de la relaxation de contraintes [34] voire d'une uniformisation du désordre.

Le dépôt d'argent se traduit par une forte diminution de $\phi_{\mathrm{p}}-\phi_{\mathrm{n}}$, de l'affinité électronique (de l'ordre de 400 à $500 \mathrm{meV}$ ) et par un ancrage de $E_{\mathrm{F}}$ plus fort. Ceci s'explique par l'apparition d'une forte densité d'états donneurs et accepteurs.

L'étude des diodes réalisées sur la surface initiale et sur les surfaces perturbées permet de déterminer les barrières finales et les facteurs d'idéalité $n$. Les résultats obtenus portés tableau II montrent que sur substrat de type $\mathrm{n}$, les barrières déduites des mesures $I(V)$ sont identiques et les coefficients $n$ sont améliorés par l'exposition à $\mathrm{O}_{2}$ ou par recuit préalable de la surface. L'émission thermoionique n'est pas perturbée 
et les mécanismes de transfert secondaires par effet tunnel ou par pièges sont diminués ce qui va dans le sens d'une interface plus abrupte avec une zone perturbée étroite localisée au plus sur quelques plans autour de l'interface et polarisée de façon à rendre compte de $\Delta \chi$ précédemment déterminé. Sur le type $\mathrm{p}$ la barrière mesurée par $I(V)$ croît sous l'action de l'oxygène tout comme le coefficient $n$. Cette croissance de la barrière, si elle peut résulter de la variation de $\Delta \chi$ permet aussi d'envisager que les états donneurs créés par l'oxygène se situent plus haut dans le gap que ceux créés par l'argent, ce qui se traduirait par l'augmentation de la barrière positive et par un remplissage plus important d'états accepteurs situés dans la partie inférieure de la bande interdite. Ces états chargés négativement qui pourraient être tout simplement des oxygènes incorporés dans la structure d'interface se comporteraient comme des relais de courant tunnel et expliqueraient la forte croissance de $n$. Le fait que les valeurs de $V_{\text {Dn }}$ obtenues par $C(V)$, dont on sait [45] qu'elles sont généralement trop élevées à cause précisément de l'existence d'une zone perturbée de surface qu'elles ne prennent pas en compte, se rapprochent des valeurs déduites des mesures de $I(V)$, va dans le sens d'une interface plus abrupte sur le type $n$. L'oxygène agirait donc, au cours d'un premier stade, comme un agent restructurateur de la surface et diminuerait ensuite la diffusion de l'argent dans le semiconducteur.

L'action du chauffage peut, quant à elle, s'analyser en termes de contraintes et de lacunes. Son action sur les défauts, tout au moins lorsqu'on se limite aux températures inférieures à $450^{\circ} \mathrm{C}$, n'est pas comparable à celle de l'oxygène. S'il modifie les topographies et les barrières, il n'efface cependant pas les défauts de clivage. Il introduit des états accepteurs et donneurs qui contribuent de façon nettement moins forte, que l'oxygène à l'ancrage de $E_{\mathrm{F}}$. Il introduit un $\Delta \chi$ négatif dont l'importance croît avec $T$ et qui correspond encore à une contribution dipolaire du type précédemment envisagé. La barrière $\phi_{\mathrm{Bn}}$ dans les diodes n'est pas modifiée sur type $\mathrm{n}$ alors que, comme précédemment, elle croît sur type p. Les facteurs d'idéalité évoluent comme dans le cas de l'exposition de $\mathrm{O}_{2}$; le fait que la barrière $V_{D_{n}}$ passe par un minimum pour $420^{\circ} \mathrm{C}$ pourrait s'interpréter en supposant que le chauffage à cette température contribuerait seulement à modifier l'ordre et les défauts en surface alors qu'une température plus élevée entraînerait une perturbation plus profonde et tendrait à la formation d'une zone perturbée plus étendue du type de celle créé par la diffusion de Ag.

\section{Conclusion.}

Les résultats que nous avons présentés nous ont permis de mettre en évidence les modifications de propriétés électroniques des surfaces (110) de GaAs induites par adsorption d'oxygène et par chauffage. Nous avons montré que leur action se traduisait par la formation d'une barrière résultant d'états induits donneurs et accepteurs et par d'importantes modifications d'affinité électronique. Les modifications de ces propriétés de surface se traduisent par des variations sensibles et comparables des caractéristiques des diodes dont l'interprétation que nous avons proposée suppose que l'interface est rendu plus abrupt par l'adsorption d'oxygène et un chauffage léger susceptible d'induire une restructuration ou d'augmenter de façon sensible la densité des lacunes.

\section{Bibliographie}

[1] Spicer, W. E., Chye, P. W., Skeath, P. R., Su, C. Y., Lindau, I., J. Vac. Sci. Technol. 16 (1979) 1422.

[2] Spicer, W. E., Lindau, I., Skeath, P. R., Su, C. Y., J. Vac. Sci. Technol. 17 (1980) 1019.

[3] Spicer, W. E., Lindau, I., Skeath, P. R., Su, C. Y., Chye, P. W., Phys. Rev. Lett. 44 (1980) 420.

[4] Williams, R. H., Varma, R. R., Montgomery, V., $J$. Vac. Sci. Technol. 16 (1979) 1418.

[5] Montmomery, V., McKinley, A., Williams, R. H., Surf. Sci. 89 (1979) 635.

[6] BaChrach, R. Z., Bauer, R. S., J. Vac. Sci. Technol. 16 (1979) 1149.

[7] Brillson, L. J., Appl. Surf. Sci. 11/12 (1982) 249.

[8] Freeouf, J. L., Appl. Phys. Lett. 41 (1982) 285.

[9] Mele, E. L., Joannopoulos, J. D., Phys. Rev. B 17 (1978) 1528.

[10] Chadi, D. J., Bachrach, R. Z., J. Vac. Technol. 16 (1979) 1159.

[11] Williams, R. H., J. Vac. Sci. Technol. 18 (1981) 929.

[12] Bolmont, D., Chen, P., Sebenne, C. A., Surf. Sci. 117 (1982) 417.
[13] Bolmont, D., Chen, P., Proix, F., Sebenne, C. A., J. Phys. C 15 (1982) 3639.

[14] Gudat, W., Eastman, D. E., J. Vac. Sci. Technol. 13 (1976) 831.

[15] Pianetta, P., Lindau, I., Gregory, P. E., Garner, C. M., SPICER, W. E., Surf. Sci. 72 (1978) 298.

[16] SPicer, W. E., Chye, P. W., Garner, C. M., Lindau, I., Pianetta, P., Surf. Sci. 86 (1979) 763.

[17] BRILlson, L. J., Surf. Sci. Rep. 2.2. (1982) 123.

[18] Chiang, S. Y., Pearson, G. L., J. Appl. Phys. 467 (1975) 2958.

[19] Akremi, A., thèse de 3e cycle Paris (1982).

[20] Smith, A., MARK, P., J. Vac. Sci. Technol. 15 (1978) 1344.

[21] Lu, Z. M., Petro, W. G., Mahowald, P. U., Oshima, M., LindaU, I., SPICER, W. E., J. Vac. Sci. Technol. $B 1$ no 3 (1983) 598. P.C.S.I. 10th Conf. Santa Fe USA (1983).

[22] Massies, J., thèse d'état Paris 1982.

[23] Massies, J., Dezaly, F., Linh, N. T., J. Vac. Sci. Technol. 17 (1980) 1134. 
[24] Barret, C., Chekir, F., Nefatti, T., Vapaille, A., Massies, J., Physica 117B-118B (1983) 851.

[25] Palau, J. M., Testemale, E., Lassabatère, L., J. Vac. Sci. Technol. 19 (1981) 192.

[26] Palau, J. M., Testemale, E., Ismail, A., Lassabatère, L., Solid State Electron. 25 (1982) 285.

[27] Lassabatère, L., Palau, J. M., Vieujot-Testemale, E., ISMAIL, A., Raisin, C., BonNET, J., SOONCKINDT, PCSI 10th Conf. Santa Fe, 1983. J. Vac. Sci. Technol. B 1 no 3 (1983) 540.

[28] W.ANG, W. I., PCSI 10th Conf. Santa Fe USA (1983). J. Vac. Sci. Technol. B 1 no 3 (1983) 574.

[29] Aspnes, D. E., HelleR, A., PCSI 10th Conf. Santa Fe USA (1983). J. Vac. Sci. Technol. B 1 no 3 (1983) 602.

[30] Ranke, W., Jacobi, K., Progress in Surf. Sci. 10 (Pergamon Press Ltd) 1981, p. 1-52.

[31] Su, C. Y., Lindau, I., Chye, P. W., Skeath, P. R., SPICER, W. E., Phys. Rev. B 15 (1982) 4045.

[32] Spicer, W. E., Pianetta, P., Lindau, I., Chye, P. W., J. Vac. Sci. Technol. 14 (1977) 885.

[33] Lindau, I., Pianetta, P., Garner, C. M., Chye, P. W., Gregory, P. R., Spicer, W. E., Surf. Sci. 63 (1977) 45.
[34] Pianetta, P., Lindau, I., Garner, C. M., Spicer, W. E., Phys. Rev. B 18 (1978) 2792.

[35] Ludeke, R., Solid State Commun. 21 (1977) 815; Phys. Rev. B 16 (1977) 5598.

[36] Brundle, C. R., Seybold, D., J. Vac Sci. Technol. 16 (1978) 1287.

[37] Mele, E. J., Joannopoulos, J. D., Phys. Rev. Lett. 341 (1978). Phys. Rev. B 18 (1978) 6998.

[38] Gregory, P. E., SPICER, W. E., Surf. Sci. 54 (1976) 229.

[39] Luth, H., Buchel, M., Dorn, R., LIEHR, M., MATZ, R., Phys. Rev. B 15 (1977) 865.

[40] Thuault, C. D., Guichar, C. M., Sebenne, C. A., Surf. Sci. 80 (1979) 273.

[41] Goldstein, B., Szostak, D. J., Ban, V. S., Surf. Sci. 57 (1976) 733.

[42] Foxon, C. T., Harvey, J. A., Joyce, B. A., J. Phys. Chem. Solid 34 (1973) 1693.

[43] Monch, W., Clemens, H. J., J. Vac. Sci. Technol. 16-5 (1979).

[44] Chen, P., Bolmont, D., Sebenne, C. A., J. Phys. C 15 (1982) 601.

[45] RHODERICK, E. H., Metal-semiconductor contacts (Oxford University Press) 1978. 